\title{
Bifurcation and Pattern Recognition
}

\author{
Yehuda Roth \\ Oranim Academic College, Kiryat Tivon, Israel \\ Email: yudroth@gmail.com
}

Received November 6, 2012; revised December 7, 2012; accepted December 15, 2012

\begin{abstract}
We propose a new approach in dealing with image recognition. We suggest that recognizing an image is related to the knowledge of a pure quantum state. Since most images are screened through incoherent photons, we introduce a method base on non-linear mapping iterations to regenerate coherence between the image photons.
\end{abstract}

Keywords: Incoherence; Bifurcation and Re-Coherence

\section{Introduction}

An image can possess a large amount of data. However, recognizing an image corresponds only with a single output. For example, face recognition describes a process for which all the face details are eventually integrated to form the single output, namely the face identification. The very fact that the face can have a single recognizing name indicates that indeed all the face details are now gathered under the same single identification.

There are mainly two physical versions for screening the same image: The frequent, for which the image emits mainly incoherent photons with a recognizable order. The photons data is then processed and integrated to form the single item recognition such as a known face. The second alternative is realized when the image is represented by a coherent light, meaning an interference pattern. In that case, all the image information is stored in a single state that according to quantum mechanics is a measurable quantity. Practically speaking, both the coherent and the incoherent photons can represent the same image although physically the representative photons' structures are entirely different.

A coherent system is defined as being composed of coordinated parts such that the whole system can be considered and analyzed as a single entity. A face, for example, is composed of many details, yet, once it is recognized it can be treated as a single person face.

Let us assume that the image is represented by a coherent-photons-state. In this paper we suggest that recognizing an image is identified with revealing the appropriate state. Thus, an image which is already presented by coherent photons is easier to analyze. Moreover, in an ideal coherent light representation, revealing the state by a single measurement is sufficient to complete the whole recognition. Unfortunately, most observed images are realized by incoherent photons and therefore this direct measurement approach seems to be unpractical. Consequently, regardless of the image representation, coherent or incoherent, the recognition process should yield a coherent state, we introduce a re-coherence formalism which is associated with some recognition device (likewise the human brain). We show in that proposed formalism that coherence or incoherence photons that arrive to the device, always terminate as a coherent state, that is a recognized image.

Roughly speaking perception is the way reality is interpreted by the individual. Image recognition falls under this category since we identify image recognition as one of the perception skills. Since perception is only an interpretation of reality, there is no necessity for the recognized state to be identical with the original state that was emitted by the image photons. For recognition purpose it is sufficient if each photon state will be constantly mapped into a same associated state. This target state will be considered as the recognition and it will be considered as the way the image is precept by the recognition device. If all recognition tools (including the human brain) act similarly, they will all agree of the same recognition even though the final result can be different from the original state. If we borrow the psychological concept to experience, we can say that when seeing an image, all individuals will experience the same vision. Surly, they might be situations for which different images will be mapped into the same state. This will be considered as the recognition limitation. To conclude, we identify the recognized state with the state mapping target rather than identifying the original state.

In this paper we propose a nonlinear mode based upon the analysis of recursive maps as is often presented in chaos theory [1]. In our approach the measuring device 
defines an external surrounding that influences the detected photons. The surrounding is represented by the recursive maps that determine the system time evolution toward coherence and consequently to the recognition. We will show the possibility of describing recursive maps with Fock space where the map time evolution can be described by means of unitary operators.

Small physical systems which are subject to the influence of the surrounding tend to become dissipative [2,3]. However, in the regular regime for which the maps converge only into few numeric values (in our model two values), we show the re-coherence effect that is needed for the recognition process as presented previously.

\section{The States Basis}

We start with the definition of the image pure state and we present the target state that is considered as the image recognition state. Later we will show how a mixed state becomes coherent under the maps iteration.

The image is is divided into an $N \times N$ small squares matrix (pixels). A pixel location is represented by the states $|i, j\rangle$, where $i, j$ describes a pixel location. With a tensor product we compose the photon states $|0\rangle|i, j\rangle$ and $|1\rangle|i, j\rangle$ as the absent and existent of a photon in the $i, j$ pixel location, respectively.

An image coherent photon is described by the following superposition

$$
|\Phi\rangle=|0\rangle \sum_{i=1}^{N} \sum_{j=1}^{N} \alpha_{i, j}|i . j\rangle+|1\rangle \sum_{i=1}^{N} \sum_{j=1}^{N} \beta_{i, j}|i . j\rangle .
$$

with $\alpha_{i, j}$ and $\beta_{i, j}$ being the superposition coefficients for the $i, j$ pixel.

In a column representation we obtain:

$$
|\Phi\rangle=\left(\begin{array}{c}
\langle 0 \mid \Phi\rangle \\
\langle 1 \mid \Phi\rangle
\end{array}\right)=\sum_{i=1}^{N} \sum_{j=1}^{N}|i, j\rangle\left(\begin{array}{c}
\alpha_{i, j} \\
\beta_{i, j}
\end{array}\right)
$$

where for a single photon we obtain,

$$
1=\langle\Phi \mid \Phi\rangle=\sum_{i=1}^{N} \sum_{j=1}^{N}\left(\left|\alpha_{i, j}\right|^{2}+\left|\beta_{i, j}\right|^{2}\right)
$$

\section{The Recognition Operator}

Sharp (or definite) recognition is associated with distinguish ability between the images where the representative states are distinguishable only when they are orthogonal. The maximum number of orthogonal states forms the space basis of states. Clearly, sharp recognition can be implemented only for a specific spanning set basis of states where the selection between the various set possibilities is subject to the observer determination [4,5]. However, unlike the measurement collapse phenomena for which the measurement output corresponds with the collapse linearity breaking [6-11], in our device an initial state experiences discrete rotations until it reaches a recognized state. This rotation will be related to the recognition mapping.

We define each pixel with a unitary operator $\hat{u}_{i, j}^{(n)}$ that rotates the coefficient vector $\left(\begin{array}{c}\alpha_{i, j}^{(n)} \\ \beta_{i, j}^{(n)}\end{array}\right)$. Applying the operator $\hat{u}_{i, j}^{(n)}$ several times generates a recursive relation as indicated by the extra superscript $(n)$. This operator generate each pixel vector with a recursive relation,

$$
\left(\begin{array}{c}
\alpha_{i, j}^{(n+1)} \\
\beta_{i, j}^{(n+1)}
\end{array}\right)=\hat{u}_{i, j}^{(n)}\left(\begin{array}{c}
\alpha_{i, j}^{(n)} \\
\beta_{i, j}^{(n)}
\end{array}\right)
$$

The unitary operator $\hat{u}_{i, j}^{(n)}$ conserve the coefficients vector norm, that is for a single photon,

$$
\forall n \quad\left|\alpha_{i, j}^{(n)}\right|^{2}+\left|\beta_{i, j}^{(n)}\right|^{2}=1
$$

The global unitary operator $\hat{U}^{(n)}$ is:

$$
\hat{U}^{(n)}=\sum_{i, j} \hat{u}_{i, j}^{(n)}|i, j\rangle\langle i, j|
$$

Applying the operator $\hat{U}^{(n)}$ on the image state $|\Phi\rangle^{(n)}$ (Equation (2) with an addition superscript $(n)$ ) we obtain

$$
\hat{U}^{(n)}|\Phi\rangle^{(n)}=\sum_{i, j}|i, j\rangle \hat{u}_{i, j}^{(n)}\left(\begin{array}{c}
\alpha_{i, j}^{(n)} \\
\beta_{i, j}^{(n)}
\end{array}\right) .
$$

Using Equation (4) we obtain,

$$
\hat{U}^{(n)}|\Phi\rangle^{(n)}=\sum_{i, j}|i, j\rangle\left(\begin{array}{c}
\alpha_{i, j}^{(n+1)} \\
\beta_{i, j}^{(n+1)}
\end{array}\right) .
$$

Defining $|\Phi\rangle^{(n+1)}=\sum_{i, j}|i, j\rangle\left(\begin{array}{c}\alpha_{i, j}^{(n+1)} \\ \beta_{i, j}^{(n+1)}\end{array}\right)$ yields

$$
\hat{U}^{(n)}|\Phi\rangle^{(n)}=|\Phi\rangle^{(n+1)}
$$

It is easy to see that the transformation conserve the state norm that is for a single photon, $\forall n,{ }^{(n)}\langle\Phi \mid \Phi\rangle^{(n)}=1$.

\section{A Simple Demonstration of a Recursive Maps}

Let us demonstrate our recognition algorithm with the following unitary maps:

$$
\hat{u}_{i, j}^{(n)}=\left(\begin{array}{cc}
\cos \theta_{i, j}^{(n)} & \sin \theta_{i, j}^{(n)} \\
-\sin \theta_{i, j}^{(n)} & \cos \theta_{i, j}^{(n)}
\end{array}\right)
$$

We define the relations 


$$
\begin{aligned}
& \alpha=|\alpha| \mathrm{e}^{\mathrm{i} a} \\
& \beta=|\beta| \mathrm{e}^{\mathrm{i} b},
\end{aligned}
$$

to obtain

$$
\begin{aligned}
\left|\alpha_{i, j}^{(n+1)}\right|^{2} & =\cos ^{2}\left(\theta_{i, j}^{(n)}\right)\left|\alpha_{i, j}^{(n)}\right|^{2}+\sin ^{2}\left(\theta_{i, j}^{(n)}\right)\left|\beta_{i, j}^{(n)}\right|^{2} \\
& +\left|\alpha_{i, j}^{(n)}\right|\left|\beta_{i, j}^{(n)}\right| \sin \left(2 \theta_{i, j}^{(n)}\right) \cos \left(a_{i, j}^{(n)}-b_{i, j}^{(n)}\right) \\
\left|\beta_{i, j}^{(n+1)}\right|^{2} & =\sin ^{2}\left(\theta_{i, j}^{(n)}\right)\left|\alpha_{i, j}^{(n)}\right|^{2}+\cos ^{2}\left(\theta_{i, j}^{(n)}\right)\left|\beta_{i, j}^{(n)}\right|^{2} \\
& -\left|\alpha_{i, j}^{(n)} \| \beta_{i, j}^{(n)}\right| \sin \left(2 \theta_{i, j}^{(n)}\right) \cos \left(a_{i, j}^{(n)}-b_{i, j}^{(n)}\right)
\end{aligned}
$$

These equations describes recursive maps of the form

$$
\begin{aligned}
& x_{i, j}^{(n+1)}=f_{i, j}\left(\theta_{i, j}^{(n)}, a_{i, j}^{(n)}, b_{i, j}^{(n)} ; x_{i, j}^{(n)}, y_{i, j}^{(n)}\right) \\
& y_{i, j}^{(n+1)}=\tilde{f}_{i, j}\left(\theta_{i, j}^{(n)}, a_{i, j}^{(n)}, b_{i, j}^{(n)} ; x_{i, j}^{(n)}, y_{i, j}^{(n)}\right) \\
& x_{i, j}^{(n)}=\left|\alpha_{i, j}^{(n)}\right|^{2}, \quad y_{i, j}^{(n)}=\left|\beta_{i, j}^{(n)}\right|^{2}
\end{aligned}
$$

To simplify further this model we assume that $\forall i, j$ $\alpha_{i, j}^{(n)}$ and $\beta_{i, j}^{(n)}$ are real numbers

$$
\left(a_{i, j}^{(n)}=b_{i, j}^{(n)}=0\right)
$$

and that

$$
\theta_{i, j}^{(n)}=\theta_{i, j}^{(n)}\left(x_{i, j}^{(n)}, y_{i, j}^{(n)}\right)
$$

There are unlimited types of recursive maps that can be derived from Equations (12)-(14). We constrict our model to maps with a double period bifurcation behavior [12]. To be more specific we assume that at each pixel $(i, j)$ the maps converge into the following values:

$$
\begin{aligned}
& \tilde{x}_{i, j}^{-}=\lim _{n \rightarrow \infty} x_{i, j}^{(n)}, \\
& \tilde{x}_{i, j}^{+}=\lim _{n \rightarrow \infty} x_{i, j}^{(n+1)}
\end{aligned}
$$

with the complementary values

$$
\begin{aligned}
& \tilde{y}_{i, j}^{-}=\lim _{n \rightarrow \infty} y_{i, j}^{(n)}=1-\tilde{x}_{i, j}^{-} \\
& \tilde{y}_{i, j}^{+}=\lim _{n \rightarrow \infty} y_{i, j}^{(n+1)}=1-\tilde{x}_{i, j}^{+}
\end{aligned}
$$

Although the maps do not converge into a single output (single values for $x$ and $y$ ) it is possible to redefine a map $g_{i, j}$ which retrieves a single stable result if we define as follows,

$$
\begin{aligned}
& g_{i, j}=f_{i, j}\left(f_{i, j}\left(x_{i, j}^{(n)}\right)\right) \equiv f_{i, j} \circ f_{i, j} \\
& \tilde{g}_{i, j} \equiv\left(1-f_{i, j}\right) \circ\left(1-f_{i, j}\right),
\end{aligned}
$$

yielding

$$
\begin{aligned}
& x_{i, j}^{(n+2)}=g_{i, j}\left(x_{i, j}^{(n)}\right) \\
& y_{i, j}^{(n+2)}=\tilde{g}_{i, j}\left(y_{i, j}^{(n)}\right)
\end{aligned}
$$

where the composed map $g_{i, j}$ corresponds with a time interval which is twice the original $f_{i, j}$-time.

Under the $g_{i, j}$-map we obtain two types of sets; the firsts converge into the $\left(x_{i, j}^{-}, y_{i, j}^{-}\right)$values and the others reach the output $\left(x_{i, j}^{+}, y_{i, j}^{+}\right)$

It is easy to identify each set as follows: Suppose that we start with initial condition namely the final states $\tilde{x}_{i, j}^{-}$or $\tilde{x}_{i, j}^{+}$. Now for $n=0,1,2,3, \cdots$ the maps split into two types:

Even series iteration

$x_{i, j}^{(2 n+2)}=g_{i, j}\left(x_{i, j}^{(2 n)}\right)$

The series $x_{i, j}^{(0)}, x_{i, j}^{(2)}, x_{i, j}^{(4)}, \cdots$

$y_{i, j}^{(2 n+2)}=1-g_{i, j}\left(x_{i, j}^{(2 n)}\right)$

The series $y_{i, j}^{(0)}, y_{i, j}^{(2)}, y_{i, j}^{(4)}, \cdots$

Odd series iteration

$x_{i, j}^{(2 n+3)}=g_{i, j}\left(x_{i, j}^{(2 n)}\right)$

The series $x_{i, j}^{(1)}, x_{i, j}^{(3)}, x_{i, j}^{(5)}, \cdots$

$y_{i, j}^{(2 n+3)}=1-g_{i, j}\left(x_{i, j}^{(2 n)}\right)$

The series $y_{i, j}^{(1)}, y_{i, j}^{(3)}, y_{i, j}^{(5)}, \cdots$

where the even and odd series converge into the values $\left(\tilde{x}_{i, j}^{-}, \tilde{y}_{i, j}^{-}\right)$and $\left(\tilde{x}_{i, j}^{+}, \tilde{y}_{i, j}^{+}\right)$, respectively.

Since the two maps operate separately, we associate them with independent states $|o\rangle$ and $|e\rangle$ types, respectively.

When applying the $g, \tilde{g}$ maps (the maps with the double steps iterations) on the initial state, that is, the initial photons state that hit the recognition machine, it iterates according to the $g$ 's maps until it reaches the final values. $\left(\tilde{x}_{i, j}^{-}, \tilde{y}_{i, j}^{-}\right)$or $\left(\tilde{x}_{i, j}^{+}, \tilde{y}_{i, j}^{+}\right)$If we denote $\alpha_{i, j}^{a_{i, j}}$ and $\beta_{i, j}^{b_{i, j}}$ as the final values of the iteration.

The state

$$
|\Phi\rangle=|0\rangle \sum_{i, j} \alpha_{i, j}^{a_{i, j}}|i . j\rangle+|1\rangle \sum_{i, j} \beta_{i, j}^{b_{i, j}}|i . j\rangle
$$

is the image recognition.

\section{The Recognition Algorithm}

The following diagram summarizes the complete recognition procedure: 


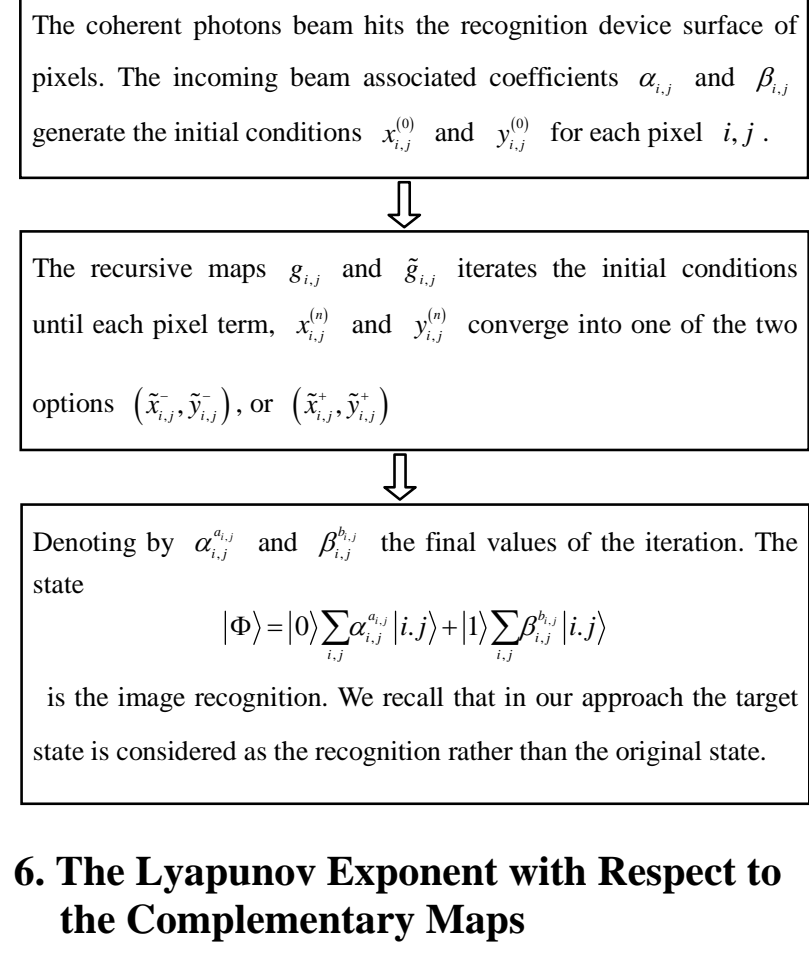

$$
|\Phi\rangle=|0\rangle \sum_{i, j} \alpha_{i, j}^{a_{i, j}}|i . j\rangle+|1\rangle \sum_{i, j} \beta_{i, j}^{b_{i, j}}|i . j\rangle
$$

is the image recognition. We recall that in our approach the target state is considered as the recognition rather than the original state.

\section{The Lyapunov Exponent with Respect to the Complementary Maps}

Earlier we mentioned that practically most images are screened by incoherent photons. Yet, in our analysis we considered only pure states. In this section we present a formalism that is responsible for a re-coherence process, that is, a formalism that generate coherence between the incoherent states. Suppose that we have the following initial conditions

$$
\begin{aligned}
& x_{i, j}^{(0)}=\tilde{x}_{i, j}^{(0)}+\Delta x_{i, j}^{(0)} \\
& y_{i, j}^{(0)}=\tilde{y}_{i, j}^{(0)}+\Delta y_{i, j}^{(0)}
\end{aligned}
$$

where the terms $\tilde{x}_{i, j}^{(0)}$ and $\tilde{y}_{i, j}^{(0)}$ are correlated such that $\tilde{x}_{i, j}^{(0)}+\tilde{y}_{i, j}^{(0)}=1$ while $\Delta x_{i, j}^{(0)}$ and $\Delta y_{i, j}^{(0)}$ represent the incoherent part of the pixels arriving photons.

According to Lyapunov theorem [13] for large $n$ we obtain

$$
\begin{aligned}
& \Delta x_{i, j}^{(n)}=\mathrm{e}^{\lambda_{x, i, j}{ }^{n}} \Delta x_{i, j}^{(0)} \\
& \Delta y_{i, j}^{(n)}=\mathrm{e}^{\lambda_{y, i, j}{ }^{n}} \Delta y_{i, j}^{(0)}
\end{aligned}
$$

where $\lambda_{x, i, j} n$ and $\lambda_{y, i, j} n$ are the Lyapunov exponents for the $x, y$ maps, obtained as:

$$
\begin{aligned}
& \lambda_{x, i, j}=\lim _{N \rightarrow \infty} \frac{1}{N} \sum_{n=0}^{N} \ln \left|\frac{\mathrm{d} \tilde{x}_{i, j}^{(n+1)}}{\mathrm{d} \tilde{x}_{i, j}^{(n)}}\right| \\
& \lambda_{y, i, j}=\lim _{N \rightarrow \infty} \frac{1}{N} \sum_{n=0}^{N} \ln \left|\frac{\mathrm{d} \tilde{y}_{i, j}^{(n+1)}}{\mathrm{d} \tilde{y}_{i, j}^{(n)}}\right| .
\end{aligned}
$$

We recall that

$$
\tilde{y}_{i, j}^{(n)}=1-\tilde{x}_{i, j}^{(n)}
$$

yielding that

$$
\frac{\mathrm{d} \tilde{x}_{i, j}^{(n+1)}}{\mathrm{d} \tilde{x}_{i, j}^{(n)}}=\frac{\mathrm{d} \tilde{y}_{i, j}^{(n+1)}}{\mathrm{d} \tilde{y}_{i, j}^{(n)}}
$$

and therefore $\lambda_{x, i, j}=\lambda_{y, i, j} \equiv \lambda_{i, j}$.

Thus we obtain

$$
\lim _{n \rightarrow \infty}\left(x_{i, j}^{(n)}+y_{i, j}^{(n)}\right)=1+\mathrm{e}^{\lambda_{i, j}{ }^{n}}\left(\Delta x_{i, j}^{(0)}+\Delta y_{i, j}^{(0)}\right) .
$$

\section{Re-Coherence and Recognition}

The Lyapunov theorem predicts a negative exponent for non-chaotic maps [13]. In particular as in our model, a negative exponent is obtained for the double period bifurcation maps type. Thus, Equation (25) yields that

$$
\lim _{n \rightarrow \infty}\left(x_{i, j}^{(n)}+y_{i, j}^{(n)}\right) \rightarrow 1
$$

that is, the incoherent terms $\Delta x_{i, j}^{(n)}, \Delta y_{i, j}^{(n)}$ vanishes during the iterations. Consequently, under the proper mapping the non-coherent photons, eventually become coherent to form the single final state. This state is now considered to stand for the image recognition state.

\section{The Recognition Efficiency}

In this paper we demonstrated that what seems to be a collapse drawback may turn into an advantage if the collapse represents the observer interpretation. The traditional approach for figuring images is by dividing them into pixels and using sophisticated algorithms in order to figure the image significance [14-17]. This corresponds to a situation with multi-pixel states. In our approach if we have a coherent image, the observer can define a quantum basis of states (which are defined as a superposition of the pixel states) rather than using the pixel basis. In quantum mechanics any quantum basis of states can be associated with a measuring device. Thus, the observer possesses the ability of building a device that measures directly the desired image. Selecting the figure basis depends on the observer determination. Consequently, the collapse can be regarded as the observer interpretation of the original coherent image. Thus, working within the image basis allows us to measure and interpret the image multi-pixel state directly in a single measurement and the result of any coherent image's multi-data state will be an interpretation into one of the device figure states.

Thus, for a coherent image, our approach is very efficient. For an incoherent image we consider the single measurement time as a very low time relative to all other recognition methods. From Equation (25) we see that the incoherent image approaches the coherent state exponentially 
with typical time of the order of $\frac{1}{\lambda_{i, j}}$.

\section{REFERENCES}

[1] H. Schanz, T. Dittrich and R. Ketzmerick, "Directed Chaotic Transport in Hamiltonian Ratchets," Physical Review E, Vol. 71, No. 2, 2005, Article ID: 026228. doi:10.1103/PhysRevE.71.026228

[2] W. H. Zurek, "Decoherence, Einselection, and the Quantum Origins of the Classical," Reviews of Modern Physics, Vol. 75, No. 3, 2003, pp. 715-775. doi:10.1103/RevModPhys.75.715

[3] W. H. Zurek, "Decoherence and the Transition from Quantum to Classical,” Physics Today, Vol. 44, No. 10, 1991, p. 36. doi:10.1063/1.881293

[4] Y. Roth, “The Quantum Observer's Consciousness,” EPL, Vol. 82, 2008, Article ID: 10006.

[5] Y. Roth, “The Observer Determination," International Journal of Theoretical Physics, Vol. 51, No. 12, 2012, pp. 3847-3855.

[6] R. Penrose, "The Road to Reality: A Complete Guide to the Laws of the Universe," Vintage Books, New York, 2004.

[7] A. Bassi, "Dynamical Reduction Models: Present Status and Future Developments," Journal of Physics: Conference Series, Vol. 67, 2007, Article ID: 012013.

[8] A. Bassi and D. G. M. Salvetti, "The Quantum Theory of Measurement within Dynamical Reduction Models," Journal of Physics A: Mathematical and Theoretical, Vol. 40, No. 32, 2007, p. 9859. doi:10.1088/1751-8113/40/32/011

[9] W. H. Zurek, "Decoherence and the Transition from Quantum to Classical,” Physics Today, Vol. 44, 1991, pp. 36-44. arXiv: quant-ph/0306072v1.

[10] S. L. Adler, et al., "Collapse Models with Non-White Noises," Journal of Physics A: Mathematical and Theoretical, Vol. 40, No. 50, 2007, pp. 15083-15098. doi:10.1088/1751-8113/40/50/012

[11] G. C. Ghirardi, A. Rimini and T. Weber, "Unified Dynamics for Microscopic and Macroscopic Systems," Physical Review D, Vol. 34, No. 2, 1986, pp. 470-491.

[12] J. D. Crawford, "Introduction to Bifurcation Theory," Reviews of Modern Physics, Vol. 63, No. 4, 1991, pp. 991-1037. doi:10.1103/RevModPhys.63.991

[13] A. Wolf, J. B. Swift, H. L. Swinney and J. A. Vastano, "Determining Lyapunov Exponents from a Time Series," Physica D, Vol. 16, No. 3, 1985, pp. 285-317. doi:10.1016/0167-2789(85)90011-9

[14] A. Y. Vlasov, 1996. arXiv:quant-ph/9703010

[15] D. Deutsch, "Quantum Theory, the Church-Turing Principle and the Universal Quantum Computer," Proceedings of the Royal Society A, Vol. 400, No. 1818, 1985, pp. 97-117. doi:10.1098/rspa.1985.0070

[16] M. A. Nielsen and I. L. Chuang, "Quantum Computation and Quantum Information,” Cambridge University Press, Cambridge, 2000.

[17] U. Fayyad, G. Piatetsky-Shapiro and P. Smyth, "From Data Mining to Knowledge Discovery in Databases," $A I$ Magazine, Vol. 17, No. 3, 1996, pp. 37-54. 